\title{
Descoberta de conhecimento com uso de técnicas de mineração de textos aplicadas em documentos textuais da investigação policial brasileira
}

\author{
Marcio Ponciano da Silva* \\ Angel Freddy Godoy Viera**
}

Artículo recibido:

17 de diciembre de 2020

Artículo aceptado:

5 de abril de 2021

Artículo de investigación
Resumo

O objetivo deste estudo é analisar como técnicas de mineração de textos aplicadas em documentos textuais da investigação policial brasileira pode promover descoberta de conhecimento. A pesquisa coletou documentos da investigação policial e submeteu ao processo de mineração de textos. O estudo utilizou as técnicas de case folding, tokenização, stopwords personalizada, bag of words e TF-IDF para extrair resultados em n-grams. Os resultados foram apresentados com word clouds. $\mathrm{Na}$ pesquisa foi usado o k-means para clusterizar os conjuntos de trigramas, identificando em cada clusters os termos mais representativos dos clusters. O uso de

* Universidade Federal de Santa Catarina, Brasil

mponcianos@gmail.com

** Universidade Federal de Santa Catarina, Centro de Ciências da Educação, Brasil a.godoy@ufsc.br INVESTIGACIÓN BIBLIOTECOLÓGICA, vol. 35, núm. 88, julio/septiembre, 2021, México, ISSN: 2448-8321 pp. 161-183 
técnicas de mineração de texto sobre esses documentos teve como propósito a extração de conhecimento não trivial. As técnicas de mineração de texto, ou descoberta de conhecimento em base de dados textual, tem a finalidade de descobrir padrões não observáveis quando analisados por manipulação humana de grande volume de documentos. Os resultados encontrados favoreceram a descoberta de conhecimentos na identificação de entidades e conexões, como também categorias temáticas da investigação.

Palavras chave: Investigação Policial; Descoberta de Conhecimento; Mineração de Textos

Descubrimiento de conocimientos mediante técnicas de minería de textos aplicadas a documentos textuales de la investigación policial brasileña

Marcio Ponciano da Silva y Angel Freddy Godoy Viera

\section{RESUMEN}

El objetivo de este estudio es analizar cómo las técnicas de minería de textos aplicadas a documentos textuales de la investigación policial brasileña pueden promover el descubrimiento de conocimiento. La investigación recopiló documentos de la investigación policial y los sometió al proceso de minería de textos. El estudio utilizó las técnicas de plegado de casos, tokenización, palabras vacías personalizadas, bolsa de palabras y TF-IDF para extraer los resultados en ngramas. Los resultados se presentaron con nubes de palabras. En la investigación, se utilizaron k-medias para agrupar los conjuntos de trigramas, identificando en cada grupo los términos más representativos de los grupos. El uso de técnicas de minería de textos en estos documentos tenía como objetivo extraer conocimientos no triviales. Las técnicas de minería de texto, o descubrimiento de conocimiento en una base de datos textual, tienen el propósito de descubrir patrones inobservables cuando se analizan mediante manipulación humana de grandes volúmenes de documentos. Los resultados encontrados favorecieron el descubrimiento de conocimientos en la identificación de entidades y conexiones, así como categorías temáticas de la investigación. 
Palabras clave: Investigación Policial; Descubrimiento del Conocimiento; Extracción de Textos

Discovery of knowledge using text mining techniques applied to textual documents of Brazilian police investigation

Marcio Ponciano da Silva and Angel Freddy Godoy Viera

\section{Abstract}

The aim of this study is to analyze how text mining techniques applied to textual documents of Brazilian police investigation can promote knowledge discovery. The research collected documents from the police investigation and submitted them to the text mining process. The study used the techniques of case folding, tokenization, custom stopwords, bag of words and TF-IDF in order to extract results in ngrams. The results were presented with word clouds. In the research, k-means were used to cluster the sets of trigrams, identifying in each clusters the most representative terms of the clusters. The use of text mining techniques on these documents was intended to extract non-trivial knowledge. The techniques of text mining, or discovery of knowledge in a textual database, have the purpose of discovering unobservable patterns when analyzed by human manipulation of large volumes of documents. The results found favored the discovery of knowledge in the identification of entities and connections, as well as thematic categories of the investigation.

Keywords: Police Investigation; Discovery of Knowledge; Text Mining

\section{INTRODUÇÃO}

Processo de descoberta de conhecimento tem sido empregado para encontrar informações ou padrões de informações, utilizando técnicas de análise e extração de dados. A descoberta de conhecimento em documentos textuais está inserida no contexto da descoberta de conhecimento em dados não estruturados. 
Este estudo coletou documentos textuais produzidos no processo de investigação policial, com propósito de submete-los a técnicas de mineração de textos para extrair conhecimento não observáveis apenas pela análise humana, devido ao volume de dados. Os resultados apresentados neste estudo fazem parte de trabalho de pesquisa de mestrado.

Esses documentos compõem volumes de inquérito policial, que no Brasil é um procedimento administrativo escrito, resultado da investigação policial e da coleta de provas com a finalidade de apurar a infração criminal. O inquérito é um instrumento que as polícias brasileiras utilizam na fase de investigação criminal para documentar a apuração de diligências produzidas em uma investigação de crime (M. Silva, 2019).

Por ano, a Polícia Federal Brasileira produz cerca de 70 mil inquéritos policiais. O somatório ao ano alcança 14 milhões de páginas de textos (Agência, 2016). Os documentos textuais do inquérito policial recebem o nome de "peças".

O objetivo geral deste estudo é investigar quais técnicas de mineração de textos oferecem melhorias à investigação policial, examinando algumas dessas técnicas e aplicando-as sobre o conjunto de documentos textuais do inquérito policial, para extrair conhecimento não trivial que possa contribuir com o processo de investigação. O estudo propõe a análise de abordagem da Ciência da Informação, por meio do uso de métodos e técnicas da Recuperação de Informação sobre as Ciências Policiais, a partir do acervo textual do inquérito policial.

Essa aproximação busca agregar conhecimento ao processo das polícias judiciárias. Borko (1968: 2) tratou essa relação simbiótica, tendo proposto que técnicas e procedimentos empregados por bibliotecários e documentaristas deveriam se basear nos resultados teóricos da Ciência da Informação. Este estudo tem aplicação de relevância social, vez que contribui com a sociedade propondo um resultado mais eficaz à investigação policial, por meio do uso de técnicas de mineração de textos.

\section{REVISÃO DA LITERATURA}

Esta pesquisa utilizou o método de pesquisa bibliográfica, elaborando uma revisão da literatura sobre o tema abordado. Essa revisão de literatura buscou principalmente trabalhos relacionados ao tema da mineração de textos.

O tema da mineração de textos está relacionado ao crescente uso de dados textuais. Segundo Zhou, Peng e Liu (2010), o enorme crescimento de documentos textuais aumentou a demanda por novos métodos de mineração de 
dados para o processamento de texto. Por isso, a mineração de textos pode se chamar de descoberta de conhecimento de bases de dados textuais (Aranha e Passos, 2006).

Como os dados de texto codificam grande parte de nosso conhecimento acumulado, eles geralmente não podem ser descartados. Como consequência, gera o acúmulo de uma grande quantidade de dados que agora estão além da capacidade de qualquer indivíduo examinar (Zhai e Massung, 2016: 4).

A descoberta de conhecimento em documentos textuais está inserida no contexto da descoberta de conhecimento em dados não estruturados. O processo de descoberta de conhecimento tem pelo menos quatro fases: a coleta de dados, o pré-processamento de dados, a mineração e o pós-processamento (L. Silva, Peres e Boscarioli, 2017: 11). A Figura 1 apresenta a ordem dessas etapas.

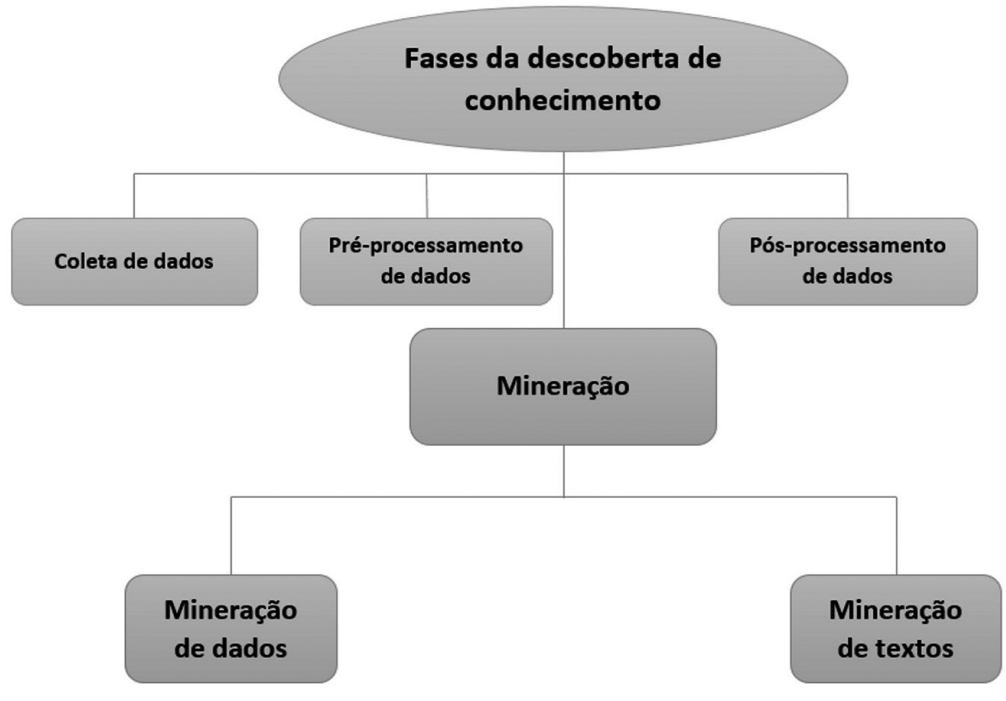

Figura 1. Etapas da mineração ou descoberta de conhecimento

A coleta de dados (textos) é a primeira etapa da mineração de textos. De acordo com Weiss et al. (2010: 8) o processo de recuperação de textos inicia com uma coleção de documentos. A etapa que se segue é o pré-processamento, onde são eliminados os fatores dependentes da linguagem para que a estrutura da linguagem se torne mais clara (Yang, Manoharan e Barber, 2014: 52).

A etapa de pré-processamento envolve a preparação dos dados antes de aplicar técnicas de mineração. $\mathrm{Na}$ preparação dos dados textuais o uso da técnica conhecida por case folding é usada para garantir a correspondência 
de strings. Essa técnica consiste em passar todas as palavras para caixa alta ou caixa baixa. Ela deve ser considerada na análise lexical (Baeza-Yates e Ribeiro-Neto, 2013: 211). Outra preparação é a remoção de acentuação. De acordo com Orengo e Huyck (2001: 188), a remoção de acentos é necessária porque há casos em que algumas formas variantes da palavra são acentuadas e outras não.

Nessa etapa, é comum o uso da tokenização, que é a primeira etapa na criação de um índice sobre qualquer tipo de dado de texto (Zhai e Massung, 2016: 61). A tokenização consiste em dividir o fluxo de caracteres em palavras, ou tokens (Weiss et al., 2010: 20). Outra técnica comum nessa etapa é a eliminação de stopwords. Segundo Baeza-Yates e Ribeiro-Neto (2013: 213), termos frequentes que aparecem em cerca de $80 \%$ dos documentos não têm utilidade para o propósito de recuperação.

Outra técnica é a redução do termo ao seu radical. Essa técnica é chamada de stemming. Para Zhai e Massung (2016: 66), stemming é o processo de reduzir uma palavra a uma forma básica. Semelhante modo, Berry (2004: 134) define como o processo que identifica a forma raiz das palavras removendo sufixos.

Um método usado nessa fase é o bag of words (BoW), que de acordo com alguns estudados (Meena e Lawrance, 2019; Costantino et al., 2017), é utilizado para extração de informações de palavras citadas em documentos. Segundo Meena e Lawrance (2019: 2610), a bag of words (BoW) constrói um vocabulário do documento fornecido.

Um modelo de ponderação de termos de um documento, muito utilizado para classificação de textos utilizado em diversos estudos é o TF-IDF (Kuang, Brantingham e Bertozzi, 2017; Meena e Lawrance, 2019). Essa medida é composta por dois cálculos, o cálculo do TF (Term Frequency) e o cálculo do IDF (Inverse Document Frequency). Esse modelo foi apontado em alguns estudos pelos bons resultados alcançados na extração de recursos (Al-Saif e Al-Dossari, 2018: 382).

Salton e McGill (1983: 205) descrevem o TF como sendo o número de vezes que um termo aparece no texto de um documento (fórmula 1):

$$
t f_{i k}=\text { freq }_{i k}
$$

O IDF mede quão rara é a palavra no corpus, conforme descrevem Manning, Raghavan e Schütze (2009: 118). Ele tem a (fórmula 2): 


$$
i d f_{t}=\log \frac{N}{d f_{t}}
$$

Esses dois elementos são a base de construção da medida resultado da multiplicação desses elementos (fórmula 3):

$$
t f i d f_{t, d}=t f_{t, d} x i d f_{t, d}
$$

Ainda nessa etapa de pré-processamento, o uso do n-gramas dá o contexto de dois (bigramas) ou três (trigramas) termos que estão próximos e que tem certa frequência que aparecem juntos. Alguns estudos atribuem ainda vantagens a métodos estatísticos como n-gramas, tendo como principais vantagens ser independente do idioma e funcionar muito bem com arquivos que contêm erros linguísticos e ruído (Al-Saif e Al-Dossari, 2018: 378).

Para apresentação de resultados, word cloud (ou nuvem de palavras) é uma técnica simples de visualização que permite a quem está analisando tenha uma compreensão de primeiro nível de conceitos/termos proeminentes (Cardoza e Wagh, 2017: 61). No contexto da mineração de textos, utilizando word cloud, quanto maior é o peso das palavras no texto a ser analisado, maior se tornam as palavras na visualização (Ramsden e Bate, 2008).

$\mathrm{Na}$ etapa de processamento um método utilizado para agrupar conjuntos de dados é a clusterização. Os algoritmos de clusterização são técnicas de aprendizado de máquina não supervisionada que agrupam um conjunto de documentos em subconjuntos e tem por objetivo criar clusters que sejam coerentes internamente, mas claramente diferentes um do outro (Manning, Raghavan e Schütze, 2009: 349).

O levantamento de trabalhos científicos que abordam o tema da mineração de textos auxilia no propósito de buscar pesquisas que possam contribuir para descoberta de conhecimento em investigações criminais. De acordo com Sampaio e Mancini (2007: 84), as revisões sistemáticas servem para incorporar um espectro maior de resultados relevantes. 
A caracterização desta pesquisa é descrita quanto a sua natureza, objetivos, abordagem e procedimentos técnicos. Quanto à natureza da pesquisa, este estudo está caracterizado por uma pesquisa aplicada. Quanto aos objetivos, trata-se de uma pesquisa exploratória. Quanto à forma de abordagem, a pesquisa trata do método misto.

Com relação aos procedimentos técnicos, este estudo se trata de pesquisa bibliográfica e documental. Ainda sobre os procedimentos técnicos deste estudo, na pesquisa bibliográfica foram consultadas as bases Web of Science, IEEE Explorer, Scopus e Lisa, além de outras fontes literárias, como os livros e leis brasileiras.

As principais etapas de uma pesquisa de mineração de textos são: coleta, pré-processamento, mineração de textos e pós-processamento. Essas etapas são descritas no modelo de descoberta de conhecimento em banco de dados (Knowledge Discovery in Databases - KDD), conhecido tradicionalmente desde 1989 (Fayyad, 2001: 32-33).

Este estudo não se propõe a esboçar diferentes modelos de processo, como CRISP-DM ou SEMMA, mas emprega etapas sob a percepção de Fayyad, onde a Mineração de Dados é uma das fases do KDD (Fayyad, Piatetsky-Shapiro e Smyth, 1996:39). A Figura 2 apresenta essas etapas com as técnicas aplicadas neste estudo.

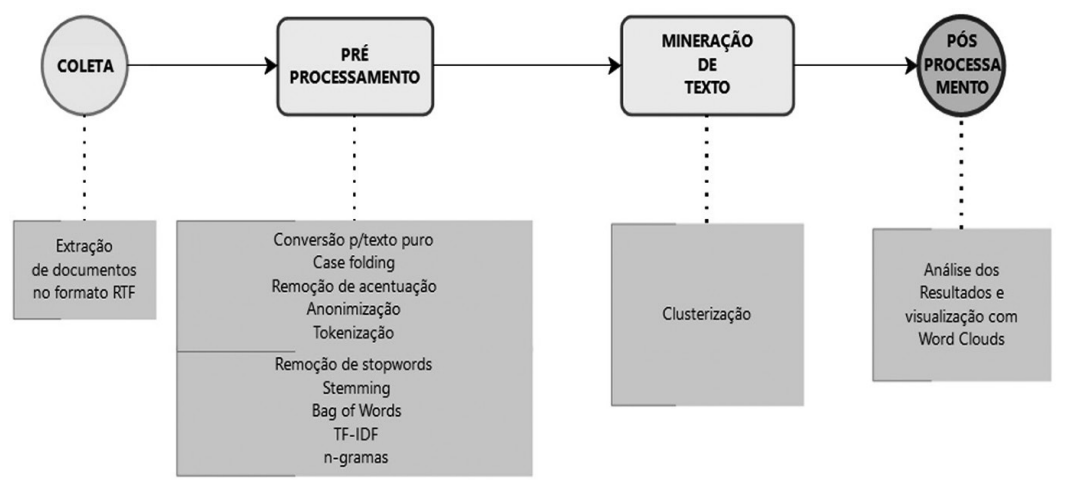

Figura 2. Etapas da mineração de textos com técnicas empregadas no estudo

Neste estudo, a coleção de 250 documentos foi coletada de um conjunto de peças textuais disponíveis em inquéritos relatados, de onde se propõe extrair conhecimento não trivial. Esses documentos foram extraídos no formato Rich Text Format (RTF). 
Ao iniciar a etapa de pré-processamento, os documentos RTF foram convertidos para o formato de texto puro, utilizando script em linguagem python escrito durante o estudo, formando um dataset único dos 250 documentos. Nesse processo foi empregada a codificação de caracteres no padrão utf-8 para garantir a compatibilidade de caracteres. Foi aplicada a técnica de case folding, sendo neste estudo convertido para letras minúsculas, além de serem removidas as acentuações de caracteres.

Esta pesquisa aplicou um processo de anonimização de dados com objetivo de salvaguardar informações de natureza pessoal. Esse processo foi divido em 9 fases. Nela forma substituídas os atributos: UF, município, CEP, telefones, nomes, RG, matrícula, placa de veículo e CPF, conforme apresentado na Figura 3.

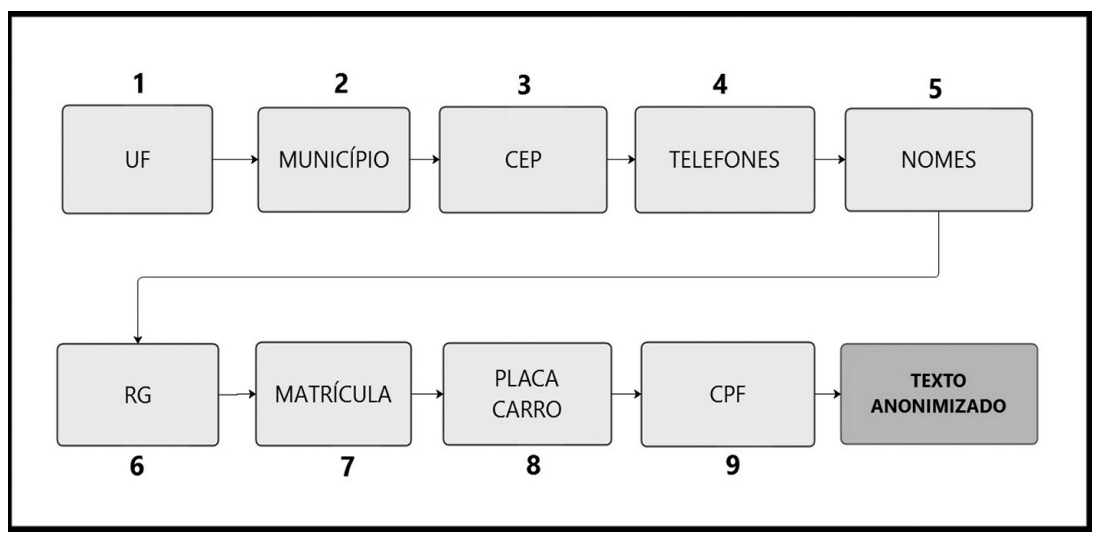

Figura 3. As 9 fases do uso da anonimização no estudo

A tokenização neste estudo foi realizada com o uso da biblioteca de código aberto do Python Natural Language Toolkit (NLTK). A biblioteca Pandas (biblioteca de código aberto do Python) foi utilizada para gerar um dataframe contendo a unidade das palavras de cada documento.

Em seguida foram eliminadas as stopwords. Além da lista de termos mais frequentes no idioma português do Brasil, foram incluídos termos frequentes no conteúdo de documentos textuais de inquérito policial.

Foram realizados dois experimentos neste estudo. $\mathrm{O}$ primeiro partiu deste ponto. Após a eliminação das stopwords, foi implementado o algoritmo de bag of words para criar um vetor booleano de frequência, indicando se cada palavra do "saco de palavras" está ou não presente no documento. Na sequência, foi aplicado o cálculo de ponderação de termos com o TF-IDF, para obter os termos mais representativos. 
No uso do TF-IDF, foi utilizada a biblioteca de código aberto do Python Scikit-Learn. O método "TfidfVectorizer" da biblioteca Scikit-Learn foi parametrizado para obter esses termos representativos no modelo $n$-grams, obtendo por meio do parâmetro "ngram_range" esses valores em unigramas, bigramas e trigramas. O emprego do TF-IDF com $n$-grams foi proposto neste estudo para descobrir tópicos, tal como proposto pelo topic model.

No segundo experimento, após a eliminação das stopwords, foi aplicada também stemming utilizando a biblioteca NLTK. O pacote stem da biblioteca NLTK utiliza vários stemmers, dentre eles o RSLPStemmer, que é específico para língua portuguesa, sendo este utilizado neste estudo. Nesse segundo caso, só após o stemming foram repetidas as aplicações de bag of words, TD-IDF e $n$-grams.

Feitos os dois experimentos, os resultados foram comparados, sendo a principal comparação os resultados de n-gramas extraídos do cálculo do TF-IDF. Com a comparação, foi escolhido um dos experimentos para utilizar na etapa de mineração de textos, que obteve resultado mais significativo.

Nessa etapa, foi utilizada a técnica de word clouds como forma de visualização dos n-gramas mais representativos. Foi elaborado script para geração da nuvem de palavras com o uso do pacote WordCloud do Python. O script elaborado utilizou outros pacotes auxiliares para gerar a imagem da nuvem de palavras, como matplotlib e numpy.

Após esses procedimentos, iniciou-se a etapa de mineração de textos propriamente dita, sendo utilizada a técnica de clusterização com o método $k$-means. $\mathrm{O}$ algoritmo de clusterização foi utilizado para agrupar conjuntos de trigramas extraídos do experimento.

Foi aplicado o Elbow Method (método do cotovelo) para determinar o número de clusters a ser utilizado no método k-means. Também foi utilizado o indicador Silhouette para análise da distância de cada cluster.

Outros métodos de agrupamentos como Agglomerative Clustering e DBSCAN são conhecidos. O Agglomerative Clustering é um método hierárquico, especialmente úteis quando o objetivo é organizar os clusters em uma hierarquia natural. O DBSCAN tem boa aplicação quando as densidades não são diferentes e precisa de uma seleção cuidadosa de parâmetros. O K-Means foi selecionado por seu uso ser menos intensivo em termos de computação, tornando mais rápido, e são adequados para conjuntos de dados muito grandes e ser próprio para agrupamento por distância entre pontos.

Com isso, foram aplicadas as principais etapas de mineração de textos, as técnicas de pré-processamento (tokenização, stopwords, stemming, BoW, TF-IDF, $n$-grams), a técnica de mineração de textos para agrupar conjuntos de dados ( $k$-means) e a técnica de pós-processamento para apresentar os 
resultados (word clouds). Após essas estapas, este estudo passou a analisar os resultados obtidos nos dois experimentos.

\section{RESUltados}

Forma obtidos resultados dos dois experimentos. Esses resultados puderam ser comparados para se verificar quais descobertas surgiram que pudessem contribuir para os objetivos deste estudo.

\section{Resultado do primeiro experimento}

O vocabulário gerado no primeiro experimento resultou em 8.274 termos (ou tokens). Com o ranqueamento dos termos mais frequentes do dataset, foram encontrados 1.049 termos que apareceram pelo menos 10 vezes nos documentos.

Com o BoW foi gerada uma matriz com esses termos mais frequentes, resultando em 250 linhas por 1.049 colunas. Cada linha representa um documento e as colunas são os termos mais frequentes do vocabulário. A Figura 4 apresenta a distribuição da frequência desses termos em cada documento.

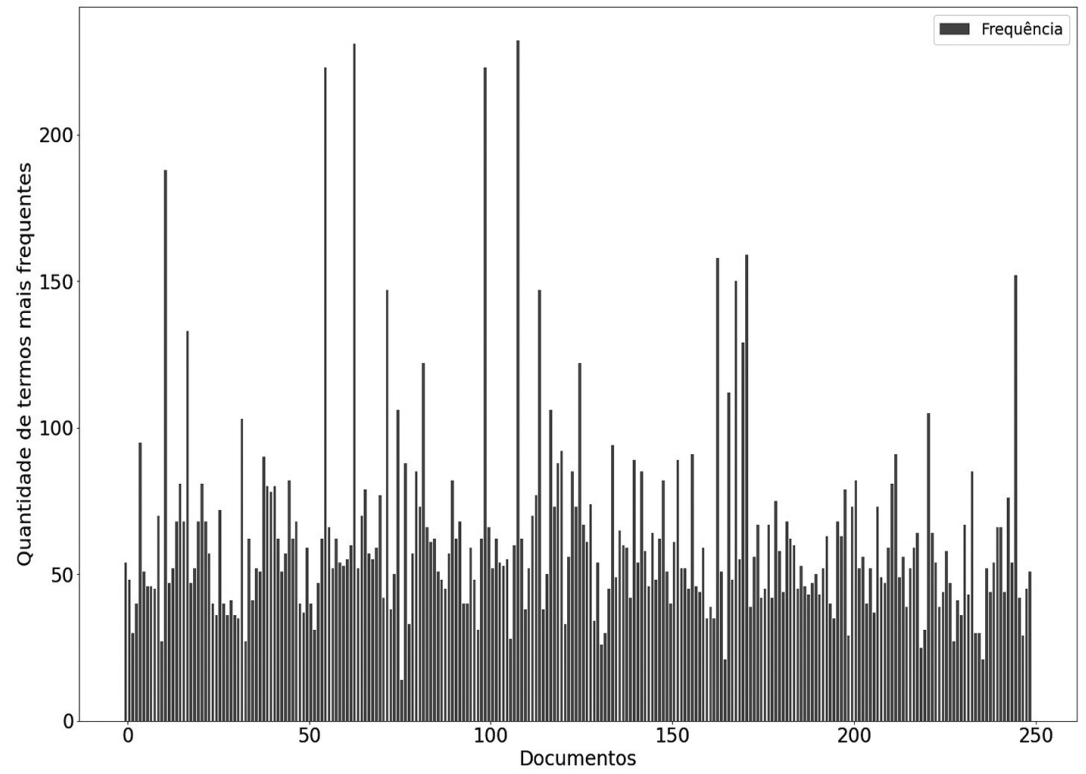

Figura 4. Distribuição da frequência dos termos do vocabulário nos documentos 
Os pesos do TF-IDF foram calculados, sendo gerada uma matriz de dispersão com 66.052 colunas (contendo unigramas, bigramas e trigramas) por 250 linhas que representam a quantidade de documentos. Uma amostra dos resultados n-gramas dessa matriz está na Figura 5.

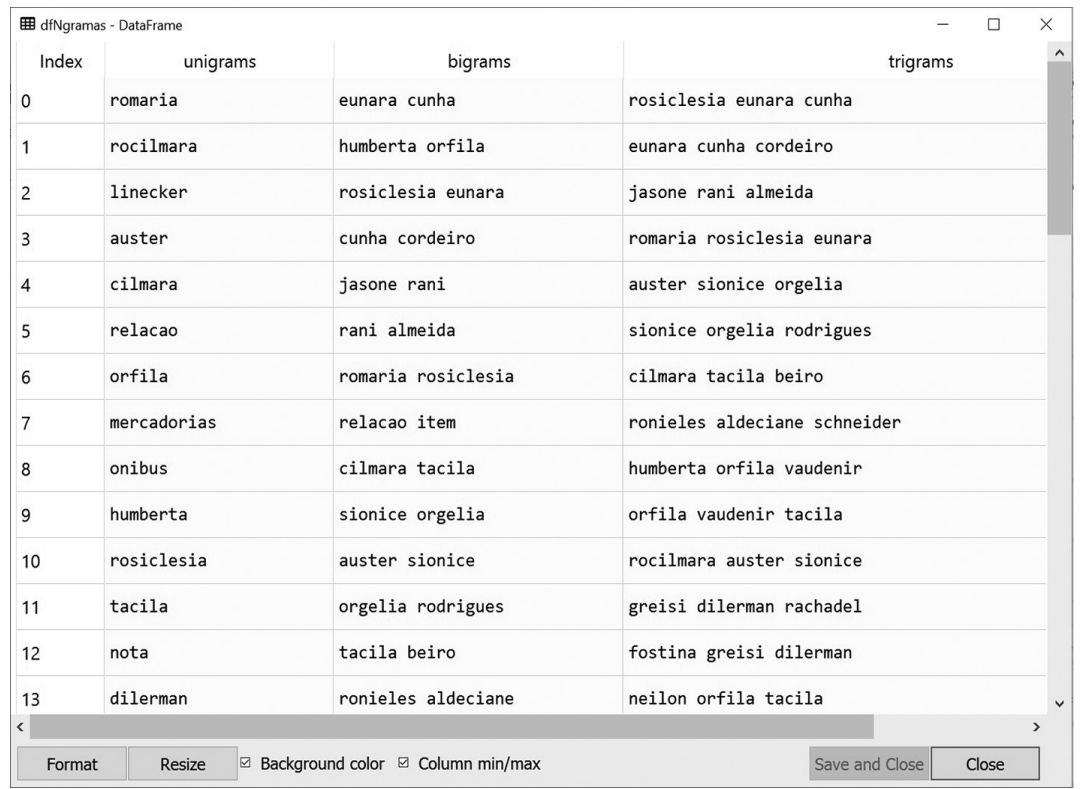

Figura 5. Dataset com n-grams extraído do TF-IDF

Essa matriz é composta com 8.274 unigramas, 27.265 brigramas e 30.513 trigramas. Este primeiro experimento será comparado com o resultado do segundo experimento.

\section{Resultado do segundo experimento}

O segundo experimento utilizou stemming para reduzir a dimensionalidade do vocabulário. Nesse experimento, o vocabulário gerado contém 5.407 termos. O ranqueamento dos termos mais frequentes do vocabulário, com frequência de pelo menos 10 ocorrências, resultou em 1.078 termos.

Neste caso, a matriz gerada com o BoW resultou em 250 linhas por 1.078 colunas. Nas linhas estão dispostos os documentos e nas colunas os termos mais frequentes do vocabulário. A distribuição da frequência desses termos está na Figura 6. 


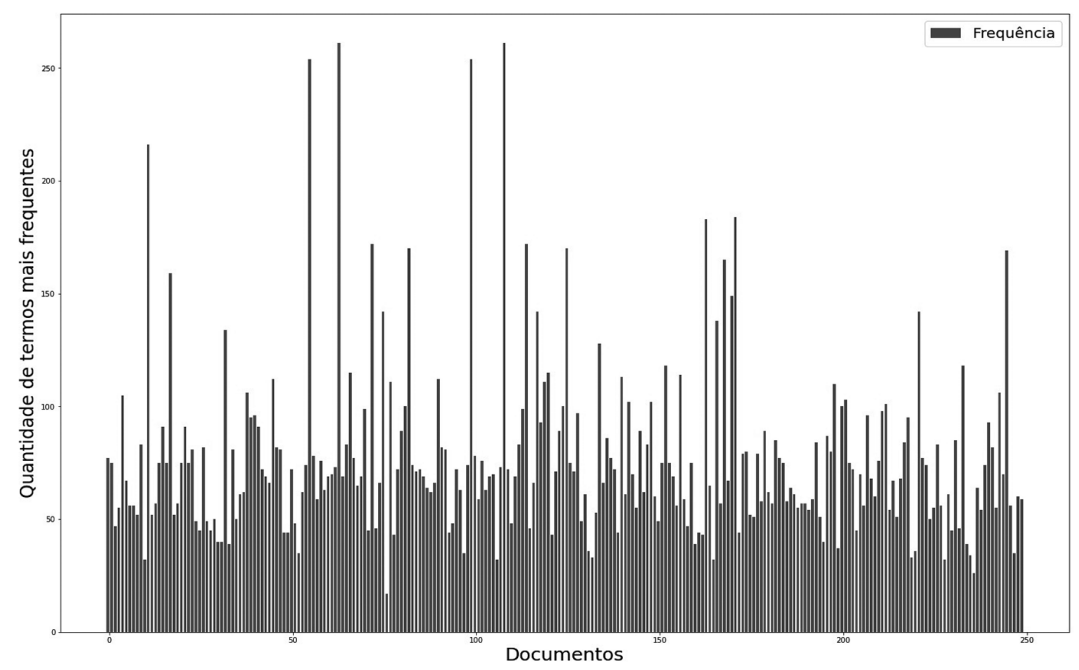

Figura 6. Distribuição da frequência dos termos do vocabulário nos documentos

A matriz de dispersão gerada para o cálculo dos pesos do TF-IDF resultou na forma de 62.064 x 250. A Figura 7 apresenta uma amostra desse conjunto de dados com o n-gramas.

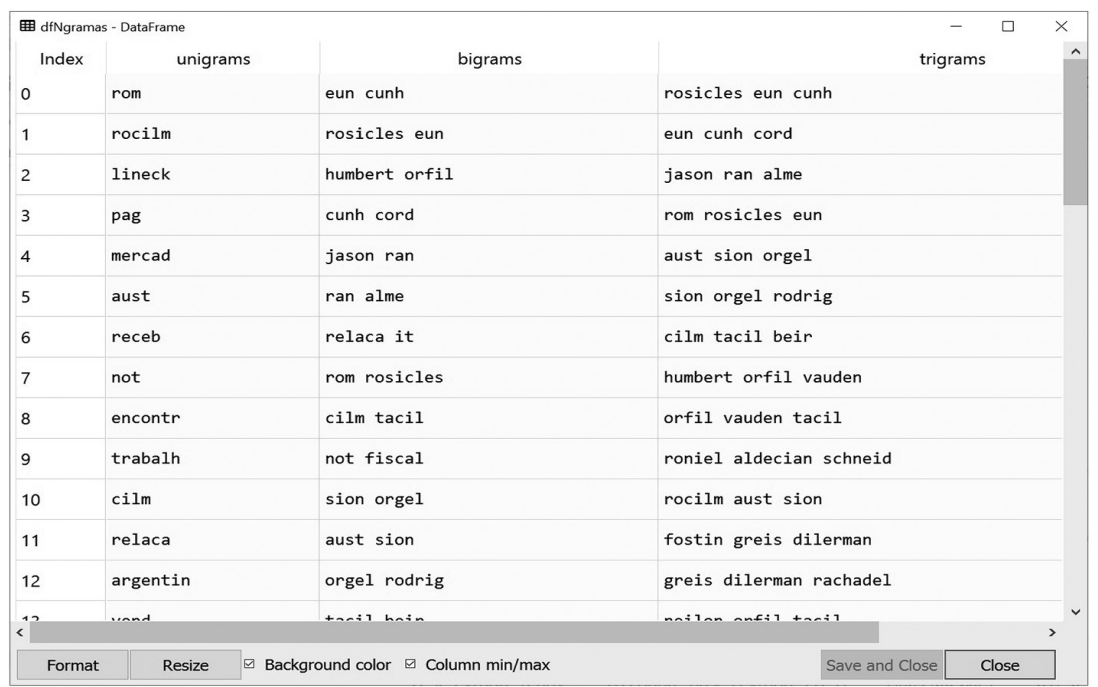

Figura 7. Dataset com n-grams extraído do TF-IDF 
Essa matriz é composta com 5.407 unigramas, 26.313 brigramas e 30.344 trigramas. A comparação deste resultado com o primeiro experimento está descrita no próximo item.

\section{Comparação dos experimentos}

Os vocabulários gerados nos dois experimentos têm uma diferença de tamanho, sendo o segundo experimento cerca de $35 \%$ menor, pois com o stemming houve uma redução da dimensionalidade dos termos do corpus. Algumas diferenças estão apresentadas no Quadro 1.

\begin{tabular}{|c|c|c|c|c|}
\hline & $\begin{array}{c}\text { Primeiro } \\
\text { experimento }\end{array}$ & $\begin{array}{c}\text { Segundo } \\
\text { experimento }\end{array}$ & Diferença \\
\hline \multicolumn{2}{|c|}{ Vocabulário } & 8.274 & 5.407 & 2.867 \\
\hline \multicolumn{2}{|c|}{ Termos com 10 ou mais ocorrências } & 1.049 & 1.078 & -29 \\
\hline \multirow{2}{*}{ Conjunto de n-gramas } & Bigramas & 27.265 & 26.313 & 952 \\
\cline { 2 - 5 } & Trigramas & 30.513 & 30.344 & 169 \\
\hline
\end{tabular}

Quadro 1. Relação de termos frequentes com o peso TF

Pode-se observar que dos termos extraídos com 10 ou mais frequências, o resultado do segundo experimento foi de uma quantidade maior de termos frequentes, apesar de ter um vocabulário menor. A quantidade de n-gramas extraídos do TF-IDF teve comportamento semelhante.

Tomando por base os trigramas extraídos do cálculo do TF-IDF, o ranqueamento dos pesos obtidos nos dois experimento foi comparado a fim de verificar se há diferença acentuada entre eles. Essa comparação determinou o resultado do experimento escolhido para utilização na etapa seguinte de processamento.

Os resultados vistos no ranqueamento foram semelhantes nos dois experimentos. Isso pode ter ocorrido em razão do tamanho do dataset utilizado. O Quadro 2 apresenta uma amostra da comparação desses resultados.

\begin{tabular}{|c|c|c|c|}
\hline \multicolumn{3}{|c|}{ Trigramas extraídos com TF-IDF } \\
\hline \multicolumn{2}{|c|}{ Primeiro Experimento } & \multicolumn{2}{c|}{ Segundo Experimento } \\
\hline rosiclesia eunara cunha & 1.258411481 & rosicles eun cunh & 1.276647444 \\
\hline eunara cunha cordeiro & 1.189530036 & eun cunh cord & 1.20725675 \\
\hline jasone rani almeida & 0.991411358 & jason ran alme & 1.004510033 \\
\hline
\end{tabular}




\begin{tabular}{|c|c|c|c|}
\hline romaria rosiclesia eunara & 0.976917018 & rom rosicles eun & 0.988558846 \\
\hline auster sionice orgelia & 0.951230086 & aust sion orgel & 0.964718881 \\
\hline sionice orgelia rodrigues & 0.939244637 & sion orgel rodrig & 0.953204469 \\
\hline cilmara tacila beiro & 0.937303188 & cilm tacil beir & 0.948106967 \\
\hline ronieles aldeciane schneider & 0.891948811 & humbert orfil vauden & 0.898771493 \\
\hline humberta orfila vaudenir & 0.885349259 & orfil vauden tacil & 0.898771493 \\
\hline orfila vaudenir tacila & 0.885349259 & roniel aldecian schneid & 0.897228426 \\
\hline rocilmara auster sionice & 0.742616682 & rocilm aust sion & 0.753424509 \\
\hline fostina greisi dilerman & 0.722217532 & fostin greis dilerman & 0.72873275 \\
\hline greisi dilerman rachadel & 0.722217532 & greis dilerman rachadel & 0.72873275 \\
\hline neilon orfila tacila & 0.697492064 & neilon orfil tacil & 0.706798274 \\
\hline orfila tacila nunes & 0.697492064 & orfil tacil nun & 0.706798274 \\
\hline canrobert sidete machado & 0.642012072 & canrobert sidet mach & 0.652673946 \\
\hline
\end{tabular}

Quadro 2. Comparação dos trigramas extraídos nos dois experimentos

Sabendo que a stemming reduz a dimensionalidade dos termos no vocabulário, a sua aplicação neste estudo não foi suficientemente expressiva. Como não houve diferença significante, a etapa da mineração de textos foi realizada com o resultado do primeiro experimento.

\section{Visualização com word cloud}

Ao gerar a visualização com nuvem de palavras apresentou os termos mais frequentes no corpus, deixando-os em evidência na "nuvem". A técnica facilitou a visualização desses termos, considerando o volume do vocabulário de trigramas.

O resultado apresentado nessa técnica contou com o ajuste da biblioteca utilizada para considerar o dicionário de trigramas. A nuvem de palavras está representada na Figura 8.

O resultado da word cloud indica a evidente valorização de nomes pelo TF-IDF. Embora não seja objetivo deste estudo o reconhecimento de entidades nomeadas (NER), essa descoberta evidenciou esse comportamento ao utilizar a ponderação da técnica TF-IDF para valorizar nomes. Com essa técnica, os termos representativos conjugam nomes e fatos, sugerindo a relação entre eles. Além de nomes, outra descoberta neste estudo é a possibilidade de estabelecer conexões com o uso do TF-IDF.

Foi visualizado grande volume de nomes presentes na nuvem de palavras. A Figura 9 apresenta outra nuvem de palavras gerada sem os nomes próprios no mesmo dicionário e considerando bigramas e trigramas. 
sabendo ler escrever

relacao item afirma

barreto rangel determina

fineia zelina campos

joenilce dilsson tavares

cynha cordeiro remito

cordeiro remiton welves

taissi lidnes inglidi

rocilmara humberta orfila

verones errmilton resert

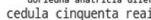

humberta orfila vaudeni

rosiclesia eunar

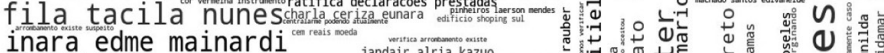
sovenir jarlesson linecker jandair alria kazuo isamo torcato jandair

remiton welves frages waster cillor romaria cleunir assuerio

sorania sayomara dilerman cleunir assuerio barbosa

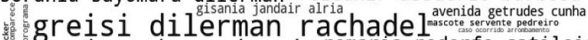

inscrito oab sob romaria rodorfo catileia

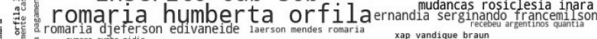

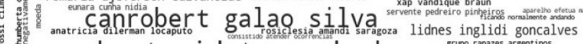

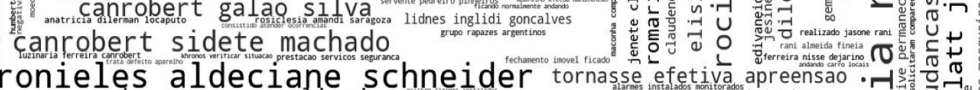

ider tornasse efetiva apreensao

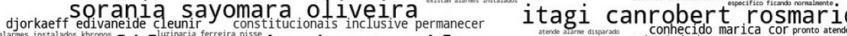

orfila vaudenir tacila

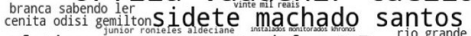

effetiva apreensao material $\mathrm{cilm}$ mar orfila cantrobert galao

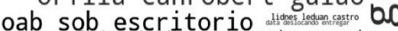

a tacila beiro

material abaixo discriminado

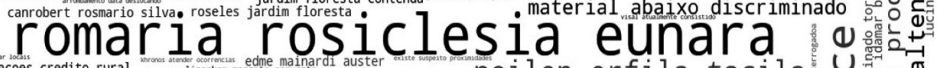

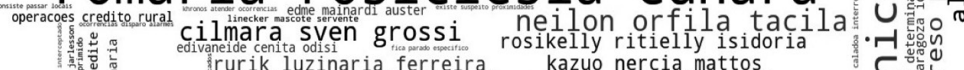

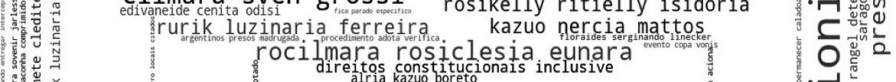

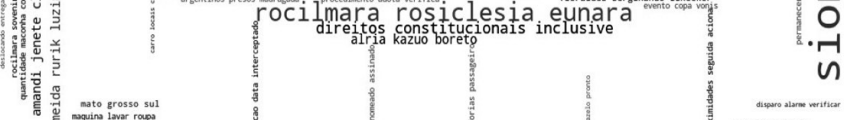

sato grosso sul
maquina lavar roppa
tacila bejico fosting.

shawan cilmara kazuo

carta precatoria extraida

auster sionice orgelia

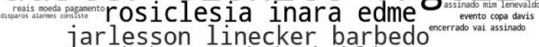

jarlesson linecker barbed

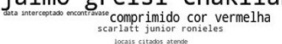

Figura 8. Termos mais representativos do dicionário de trigramas 


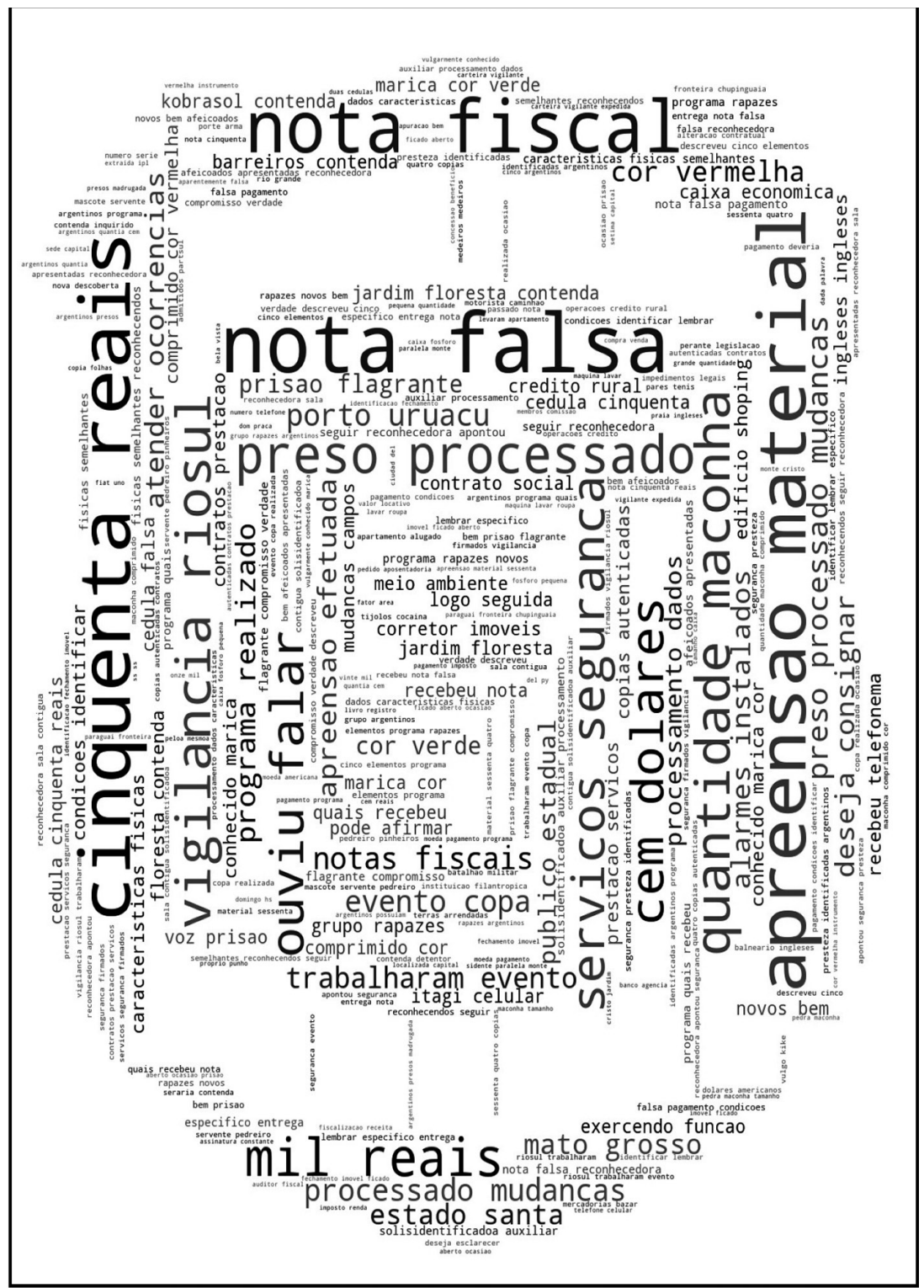

Figura 9. Termos mais representativos dos n-grams sem nomes próprios 
Com a retirada dos nomes próprios os temas abordados no contexto do dicionário surgem em destaque. Esses termos representam mais claramente o resultado do TF-IDF. A próxima etapa tentou agrupar os resultados para identificar as categorias representativas.

\section{Resultado da clusterizaçáo}

Como se viu no resultado do TF-IDF, esse método valoriza nomes. Contudo, para esta etapa foram eliminados os nomes da lista de atributos a serem utilizados na clusterização.

Para a clusterização, o conjunto de dados utilizado para treino representou $60 \%$ do dataset gerado no primeiro experimento e o conjunto para teste representou $40 \%$ do dataset. Após testar um número variado de clusters, com valor de K entre 2 e 19. A cada interação desse intervalo os textos foram classificados em clusters, com base nas features mais relevantes.

Com o resultado do Elbow Method e do Silhouette, utilizado na análise da métrica estatística da distância de cada grupo, o número de clusters foi definido em $\mathrm{k}=8$. Os valores da variável Inertia_ (do algoritmo do método do cotovelo) e a pontuação média do indicador Silhouette estão no Quadro 3.

\begin{tabular}{|c|c|c|}
\hline $\begin{array}{c}\text { Interação de } \\
\text { clusters }\end{array}$ & Valores de Inertia_ & Valores de score de Silhouette \\
\hline 2 & 26.407766542630895 & 0.21447120803450873 \\
\hline 3 & 23.40613742895484 & 0.4543829253092717 \\
\hline 4 & 21.5162601542226 & 0.533569334507583 \\
\hline 5 & 20.19718766312582 & 0.614872536807049 \\
\hline 6 & 19.119907149891077 & 0.6472925266298454 \\
\hline 7 & 17.34682388257048 & 0.7082602221754799 \\
\hline 8 & 14.755351548672904 & 0.7916165788142853 \\
\hline 9 & 14.170636026494282 & 0.8182662988835868 \\
\hline 10 & 13.449997605349845 & 0.8418286611777918 \\
\hline 11 & 12.860182294729992 & 0.8798681665203688 \\
\hline 12 & 12.5578304435155 & 0.8874530403988156 \\
\hline 13 & 11.99133960052284 & 0.9080805754423239 \\
\hline 14 & 11.65154548866381 & 0.9202136520380176 \\
\hline 15 & 11.176742884157882 & 0.9367101164812598 \\
\hline 16 & 10.942296599429019 & 0.9284410700703736 \\
\hline 17 & 10.684578800515458 & 0.9199451324245363 \\
\hline 18 & 10.497505698669887 & 0.9074891070016042 \\
\hline & &
\end{tabular}


O resultado do Elbow Method sugere uma leve diminuição na soma dos erros quadráticos das instâncias de cada cluster quando ocorre a interação com 8 clusters. O gráfico do método do cotovelo identifica visualmente o declínio desse atributo Inertia_, como na Figura 10.

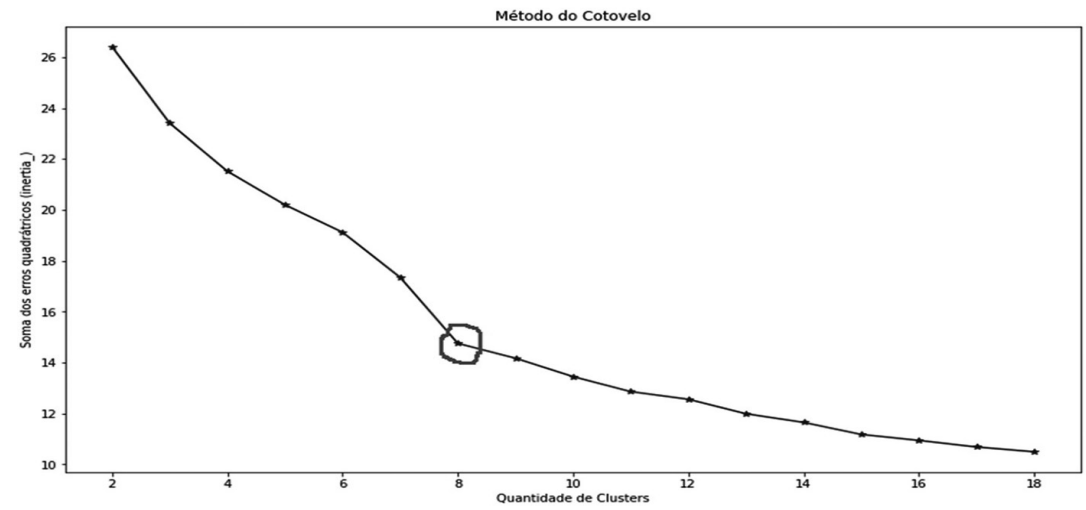

Figura 10. Gráfico do método do cotovelo

O valor de Silhouette entre 0 e 1 mostra a sobreposição. Caso o valor do Silhouette fosse próximo de 1 , seria uma indicação de classificação mais resolutiva e se o valor fosse mais próximo do 0 significa superposição dos cluster. Tendo o método do cotovelo indicado o número de 8 clusters, o valor de pontuação média do Silhouette nessa interação é uma variação razoavelmente próxima de 1 , o suficiente para definir o valor de $\mathrm{k}=8$. A Figura 11 apresenta o gráfico do Silhouette.

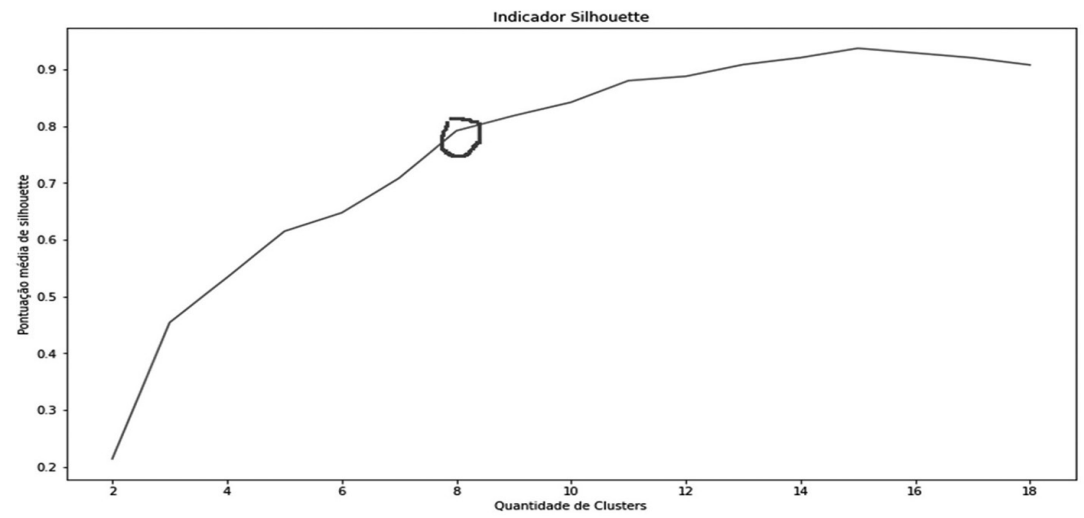

Figura 11. Gráfico do indicador Silhouette 
Com esses resultados do método do cotovelo e do indicador Silhouette, foi utilizado o agrupamento com 8 clusters. Com os clusters gerados e a análise dos termos mais representativos em cada cluster foi possível identificar categorias no agrupamento. Esses termos estão representados no Quadro 4.

\begin{tabular}{|c|c|}
\hline Cluster & Termos mais representativos nos clusters \\
\hline 0 & $\begin{array}{l}\text { notas fiscais - mil reais - apreensao efetuada - credito rural - caixa economica - } \\
\text { exercendo funcao - operacoes credito rural }\end{array}$ \\
\hline 1 & $\begin{array}{c}\text { nota falsa - prisao flagrante - quais recebeu - recebeu nota - condicoes identificar - } \\
\text { nota falsa reconhecedora - nota falsa pagamento - entrega nota falsa }\end{array}$ \\
\hline 2 & $\begin{array}{l}\text { alarmes ocorrências - alarmes instalados - vigilante - verifica arrombamento - alar- } \\
\text { me disparado - entregar interceptado }\end{array}$ \\
\hline 3 & $\begin{array}{l}\text { apreensao material - vigilancia - prestacao servicos seguranca - seguranca firmados } \\
\text { seguranca - servico seguranca }\end{array}$ \\
\hline 4 & $\begin{array}{l}\text { quantidade maconha - cor vermelha - cor verde - maconha comprimido cor - compri- } \\
\text { mido cor - comprimido cor vermelha }\end{array}$ \\
\hline 5 & $\begin{array}{l}\text { grupo rapazes - grupo argentinos - dolares americanos - cem dolares - rapazes } \\
\text { argentinos - pagamento deveria }\end{array}$ \\
\hline 6 & $\begin{array}{c}\text { cinquenta reais - cedula cinquenta - cedula cinquenta reais - contenda detentor - } \\
\text { numero serie - recebido cedula }\end{array}$ \\
\hline 7 & $\begin{array}{l}\text { programa realizado - pagamento programa - moeda pagamento - pedra maconha - } \\
\text { pedra maconha tamanho - maconha tamanho - cem reais - reais moeda }\end{array}$ \\
\hline
\end{tabular}

Quadro 4. Termos mais representativos em cada cluster

Os termos mais relevantes dos clusters estão relacionados com áreas de atuação da Polícia Federal brasileira. Eles estão indicados juntamente com sua área respectiva, conforme o Quadro 5.

\begin{tabular}{|c|c|}
\hline Cluster & Área de atuação \\
\hline 0 & Corrupção \\
\hline 1 & Fazendária \\
\hline 2 & Fiscalização de Segurança Privada \\
\hline 3 & Defesa Institucional \\
\hline 4 & Tráfico de Entorpecentes \\
\hline 5 & Controle Migratório \\
\hline 6 & Financeiro \\
\hline 7 & Tráfico Internacional de Entorpecentes \\
\hline
\end{tabular}


Como os algoritmos de aprendizado não supervisionado não utilizam categorias, essas dependem de análise. O Quadro 5 apresenta as categorias identificadas na análise de cada cluster, com base dos termos mais relevantes.

\section{CONSIDERAÇões FINAIS}

Este estudo teve o objetivo de descobrir conhecimento não trivial em documentos textuais da investigação policial brasileira. Para isso, utilizou técnicas de mineração de textos, buscando identificar os termos mais representativos do corpus.

Os resultados desta pesquisa constituíram as bases para compreensão de como as técnicas de mineração de textos em documentos textuais do inquérito policial podem auxiliar à investigação policial brasileira. O emprego das técnicas e métodos utilizados permitiram descobrir características dos documentos da investigação policial, tais como os termos mais relevantes de um conjunto de documentos.

A análise de diversos documentos textuais de inquéritos policiais diferentes permitiu o estudo comparativo de documentos que tratam temas diversos. O uso de técnicas para descoberta de conhecimento resultou na identificação das categorias desses documentos. $\mathrm{Na}$ avaliação desses resultados obtidos, essas categorias foram identificadas como temas de atuação da Polícia Federal brasileira.

Além das técnicas de descoberta de conhecimento indicarem categorias de atuação da polícia, verificando os termos mais representativos em documentos, elas também apresentaram bons resultados para identificar nomes e conexões com fatos. Os termos mais representativos servirão de análise sobre quais áreas concentram mais volume, de acordo com o escopo do universo da amostra.

Este estudo recomenda a aplicação das técnicas nele utilizadas em amostra com maior volume documentos textuais e maior amplitude de casos. Novos estudos podem aproveitar as técnicas e as bibliotecas utilizadas nesta pesquisa e também fazer uso de outras técnicas de mineração de textos.

\section{REFERÊNCIAS}

Agência de Notícias - Polícia Federal. 2016. "Polícia Federal lança sistema de inquérito eletrônico”. Acao, Divisão de Comunicação Social, 24 de outubro. Acessado 14 de dezembro de 2020.

http://www.pf.gov.br/agencia/noticias/2016/10/policia-federal-lanca-sistema-de-inquerito-eletronico 
Al-Saif, Hissah, e Hmood Al-Dossari. 2018. "Detecting and classifying crimes from arabic twitter posts using text mining techniques". International Journal of Advanced Computer Science and Applications 9 (10): 377-387. https://doi.org/10.14569/IJACSA.2018.091046

Aranha, Christian, e Emmanuel Passos. 2006. "A Tecnologia de Mineração de Textos”. Revista Eletrônica de Sistemas de Informação 5 (2): 1-8. https://doi.org/10.21529/RESI.2006.0502001

Baeza-Yates, Ricardo, e Berthier Ribeiro-Neto. 2013. Recuperação de Informação: Conceitos e Tecnologia das Máquinas de Busca. 2a. ed. Traduzido por Leandro Krug Wives e Viviane Pereira Moreira. Brasil: Bookman.

Berry, Michael W., org. 2004. Survey of Text Mining. New York, NY: Springer New York. https://doi.org/10.1007/978-1-4757-4305-0

Borko, Harold. 1968. "Ciência da informação: o que é isto?". American Documentation: 1-6. Tradução livre. Acessado 14 de dezembro de 2020. https://edisciplinas.usp.br/pluginfile.php/2532327/mod_resource/content/1/ Oque\%C3\%A9CI.pdf

Cardoza, Clinton, e Rupali Wagh. 2017. "Text analysis framework for understanding cyber-crimes". International Journal of Advanced and Applied Sciences 4 (10): 58-63. https://doi.org/10.21833/ijaas.2017.010.010

Costantino, Gianpiero, Antonio La Marra, Fabio Martinelli, Andrea Saracino, e Mina Sheikhalishahi. 2017. "Privacy-preserving text mining as a service". IEEE Symposium on Computers and Communications (ISCC), 890-897. https://doi.org/10.1109/ISCC.2017.8024639

Fayyad, Usama. 2001. "Knowledge Discovery in Databases: An Overview”. In Relational Data Mining, organizado por Sašo Džeroski e Nada Lavra , 28-47. Berlin, Heidelberg: Springer Berlin Heidelberg. https://doi.org/10.1007/978-3-662-04599-2_2

Fayyad, Usama, Gregory Piatetsky-Shapiro, e Padhraic Smyth. 1996. "From Data Mining to Knowledge Discovery in Databases”. AI Magazine 17 (3): 37-54. https://doi.org/10.1609/aimag.v17i3.1230

Kuang, Da, P. Jeffrey Brantingham, e Andrea L. Bertozzi. 2017. "Crime topic modeling". Crime Science 6 (1). https://doi.org/10.1186/s40163-017-0074-0

Manning, Christopher D., Prabhakar Raghavan, e Hinrich Schütze. 2009. Introduction to Information Retrieval. UK: Cambridge University Press.

Meena, K., e Raj Lawrance. 2019. "An automatic text document classification using modified weight and semantic method". International Journal of Innovative Technology and Exploring Engineering 8 (12): 2608-2622. https://doi.org/10.35940/ijitee.K2123.1081219

Orengo, Viviane Moreira, e Christian Huyck. 2001. "A Stemming Algorithm for the Portuguese Language". Proceedings Eighth Symposium on String Processing and Information Retrieval, 186-193. Laguna de San Rafael, Chile: IEEE. https://doi.org/10.1109/SPIRE.2001.989755

Ramsden, Andrew, e Andrew Bate. 2008. "Using Word Clouds in Teaching and Learning”, agosto. https://researchportal.bath.ac.uk/en/publications/using-word-clouds-in-teaching-and-learning 
Salton, Gerard, e Michael J. McGill. 1983. Introduction to Modern Information Retrieval. USA: McGraw-Hill, Inc.

Sampaio, Rosana Ferreira, e Marisa Cotta Mancini. 2007. "Estudos de revisão sistemática: um guia para síntese criteriosa da evidência científica”. Revista Brasileira de Fisioterapia 11 (1): 83-89. https://doi.org/10.1590/S1413-35552007000100013

Silva, Leandro, Sarajane Peres, e Clodis Boscarioli. 2017. Introdução a Mineração de Dados com aplicações em R. Rio de Janeiro: Elsevier.

Silva, Marcio Ponciano da. 2019. "Focos de inovação na Polícia Federal para combater a corrupção e o crime organizado". In Carreiras típicas de Estado: desafios e avanços na prevenção e no combate à corrupção, 275-284. Belo Horizonte: Fórum.

Weiss, Sholom Menachem, Nitin Indurkhya, Tong Zhang, e Fred Damerau. 2010. Text Mining: Predictive Methods for Analyzing Unstructured Information. New York: Springer.

Yang, Yongpeng, Monisha Manoharan, e Kathleen Suzanne Barber. 2014. "Modelling and Analysis of Identity Threat Behaviors through Text Mining of Identity Theft Stories". IEEE Joint Intelligence and Security Informatics Conference, 50-63. https://doi.org/10.1109/JISIC.2014.35

Zhai, ChengXiang, e Sean Massung. 2016. Text Data Management and Analysis: A Practical Introduction to Information Retrieval and Text Mining. USA: Association for Computing Machinery and Morgan \& Claypool.

Zhou, Xuezhong, Yonghong Peng, e Baoyan Liu. 2010. "Text mining for traditional Chinese medical knowledge discovery: A survey". Journal of Biomedical Informatics 43 (4): 650-660.

https://doi.org/10.1016/j.jbi.2010.01.002

Para citar este texto:

Silva, Marcio Ponciano da e Angel Freddy Godoy Viera. 2021. "Descoberta de conhecimento com uso de técnicas de mineração de textos aplicadas em documentos textuais da investigação policial brasileira”. Investigación Bibliotecológica: archivonomía, bibliotecología e información 35 (88): 161-183. http://dx.doi.org/10.22201/iibi.24488321xe.2021.88.58389 